\title{
Early Initiation of Veno-venous Extracorporeal Membrane Oxygenation in the Emergency Room as A Salvage Treatment for Patients with Coronavirus Disease 2019 with Severe Acute Respiratory Distress Syndrome
}

\author{
Kenta Aida ${ }^{1} \star$, Kazunari Azuma ${ }^{1}$, Kentaro Morinaga ${ }^{1}$, Yuki Nishiyama ${ }^{1}$, \\ Kazuma Kimura ${ }^{1}$, Jun Oda ${ }^{1}$
}

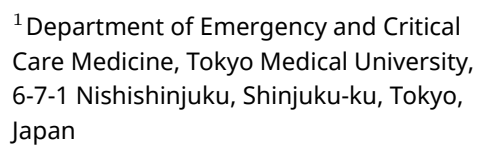

${ }^{1}$ Department of Emergency and Critical Care Medicine, Tokyo Medical University, 6-7-1 Nishishinjuku, Shinjuku-ku, Tokyo, Japan

\section{*Correspondence}

kenta-a@tokyo-med.ac.jp

(Kenta Aida)

\begin{abstract}
Background: Since veno-venous extracorporeal membrane oxygenation (VV-ECMO) is highly invasive and may be used over a long period of time, its introduction is usually carefully considered and planned. Here, we report the case of a life-saving procedure by introducing VV-ECMO in the emergency room. Case presentation: A 56 year-old man had fatigue for 3 days along with rapidly worsening dyspnea. On arrival at the emergency room, he presented with severe hypoxemia. Tracheal intubation and mechanical ventilation were immediately initiated; however, hypoxemia persisted. Therefore, VV-ECMO was introduced in the emergency room. The patient's respiratory condition gradually improved, and VV-ECMO was terminated on hospital day 6. Conclusion: The early introduction of VV-ECMO in the emergency room was effective against acute respiratory distress syndrome caused by coronavirus disease 2019. Further studies should be performed to confirm the efficacy of early VV-ECMO introduction.
\end{abstract}

\section{Keywords}

Extracorporeal membrane oxygenation, COVID-19, Severe acute respiratory syndrome coronavirus 2, Critical care

\section{Background}

Veno-venous extracorporeal membrane oxygenation (VVECMO) is the ultimate symptomatic treatment for patients with severe respiratory failure. However, it is an invasive procedure and may be employed for long-term use; therefore, its introduction is usually carefully considered. Only few reports have demonstrated the early introduction of VV-ECMO in the emergency room (ER), especially for the management of patients with coronavirus disease 2019 (COVID-19) who have severe acute respiratory distress syndrome (ARDS). Herein, we report the case of a patient with COVID-19 who suddenly developed ARDS and who could reintegrate with society after the early introduction of VV-ECMO in the ER.

\section{Case presentation}

A 56 year-old man had fatigue for 3 days. He developed dyspnea that showed rapid exacerbation 3 - $4 \mathrm{~h}$ after its onset. Moreover, he was transported to our ER. The patient had a history of benign prostatic hyperplasia and was a smoker (one pack per day) for $>20$ years. His body mass index was 18.9 $\mathrm{kg} / \mathrm{m}^{2}$ (height: $172 \mathrm{~cm}$; weight: $56 \mathrm{~kg}$ ).

Vital signs at the ER were as follows: Glasgow coma scale,
E4V4M6; respiratory rate, 38 breaths per min; oxygen saturation, $70 \%$ under oxygen mask at $15 \mathrm{~L} / \mathrm{min}$; heart rate, 104 beats per min; and blood pressure, 144/93 mmHg. Physical examination revealed bilateral wheezing. Chest radiography and computed tomography showed bilateral ground-glass opacities and consolidation (Fig. 1). Transthoracic echography showed preserved left ventricular function and no valvular disease.

On arrival at our ER, significant respiratory distress was evident with an increased respiratory rate of 38 breaths per min and low oxygen saturation of $70 \%$. His arterial blood gas was $\mathrm{pH} 7.40$, partial pressure of carbon dioxide $\left(\mathrm{PaCO}_{2}\right)$ was $32.5 \mathrm{mmHg}$, and partial pressure of oxygen $\left(\mathrm{PaO}_{2}\right)$ was $50.7 \mathrm{mmHg}$. He required intubation and mechanical ventilation; however, it took $1 \mathrm{~h}$ to obtain consent. With a rate of 22 beats per minute on the pressure control ventilation, an inspiratory pressure of $28 \mathrm{~cm} \mathrm{H}_{2} \mathrm{O}$, a positive end-expiratory pressure of $20 \mathrm{~cm} \mathrm{H}_{2} \mathrm{O}$, and a fraction of inspired oxygen $\left(\mathrm{FiO}_{2}\right)$ of $100 \%$ generating, tidal volumes of $260 \mathrm{ml}$ are generated. Although lung-protective ventilation strategies were adopted, a blood gas test conducted $40 \mathrm{~min}$ after the mechanical ventilation showed $\mathrm{pH}$ of $7.36, \mathrm{PaO}_{2}$ of $62.6 \mathrm{mmHg}$, and $\mathrm{PaCO}_{2}$ of $40.4 \mathrm{mmHg}$, indicating poor improvement of hypoxemia. To prevent cardiac arrest due to hypoxemia, VV- 


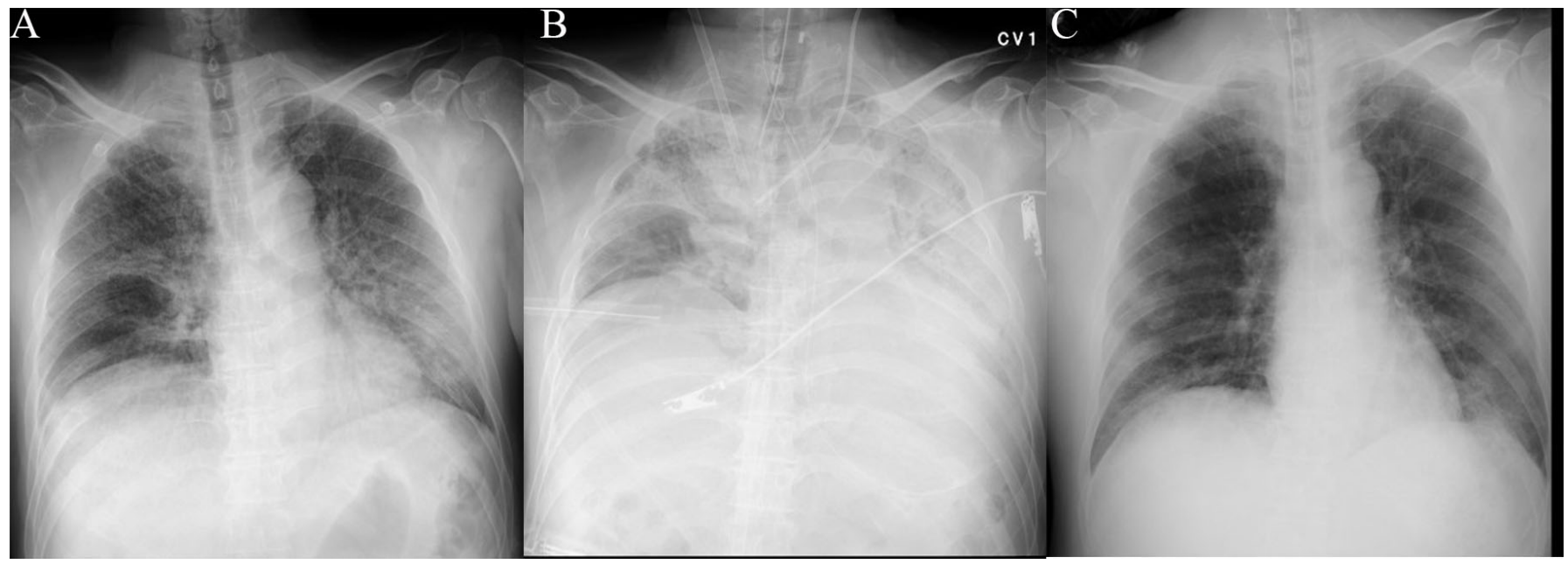

F I G U R E 1. Chest X-ray of a 56-year-old man with COVID-19.

(A) On arrival at ER. Ground-glass opacities are apparent in both lobes.

(B) After VV-ECMO cannulation in ER. Bilateral pulmonary infiltrates have progressed.

(C) Day 6; after removal of VV-ECMO. Bilateral pulmonary infiltrates have improved.

VV-ECMO: veno-venous extracorporeal membrane oxygenation.

ECMO was introduced in the ER 90 min after mechanical ventilation. Vascular puncture was performed using echo and fluoroscopy. After the introduction of VV-ECMO, the ventilator was set to lung rest. Anticoagulation therapy with intravenous unfractionated heparin infusion targeting an activated partial thromboplastin time (APTT) of 35 - $60 \mathrm{~s}$ was initiated, and the patient was admitted to the emergency and intensive care unit. The time course in the ER is summarized in Table 1. Ventilator parameters and arterial blood gas analyses are shown in Table 2.

T A B L E 1. Summary of the timeline of events in the emergency room.

$\begin{array}{cc}\text { Time } & \text { Event } \\ 0: 30 & \text { Emergency call } \\ 1: 15 & \text { Arrival at the emergency room } \\ 1: 20 & \text { Chest X-ray } \\ 1: 25 & \text { Chest CT } \\ 1: 30 & \text { Explaining the medical condition and the treatment } \\ 2: 30 & \text { Tracheal intubation } \\ 4: 00 & \text { VV-ECMO } \\ 6: 00 & \text { Admitted to the emergency and intensive care unit }\end{array}$

VV-ECMO: veno-venous extracorporeal membrane oxygenation; CT: computed tomography.

Based on the clinical course and imaging tests, the patient was found to have severe ARDS caused by COVID19; thus, methylprednisolone ( $1 \mathrm{mg} / \mathrm{kg} / \mathrm{day})$ was administered on hospital day 1. On hospital day 4, the patient tested positive for severe acute respiratory syndrome coronavirus 2 (SARS-CoV-2) using polymerase chain reaction. At that time, favipiravir was the only antiviral drug available in our hospital for the treatment of COVID-19; remdesivir was not available. Therefore, favipiravir was administered for 2 weeks.
His respiratory status gradually improved, and VV-ECMO was removed on hospital day 6 (Fig. 1). The administration of unfractionated heparin was continued for thromboprophylaxis. After the removal of ECMO, ventilator-associated pneumonia, sputum suffocation, and atelectasis were frequently observed. However, the respiratory status gradually improved with antibiotics, multiple bronchoscopic sputum aspiration, and prone-positioning therapy. The respirator was removed after performing tracheostomy on hospital day 46, and the patient was transferred to the general ward on hospital day 51. Motor function and muscle weakness occurred; however, they improved with rehabilitation, allowing the patient to walk independently. The patient was discharged on hospital day 79 , and he reintegrated in society with normal neurological examination results.

\section{Discussion}

This report describes the early initiation of VV-ECMO in the ER to save a patient with COVID-19 who developed rapidly progressing and severe ARDS. The effect of VV-ECMO on ARDS has been reported to be limited in case of long-term management with high airway pressure and oxygen concentration [1]. However, there is no consensus on the effectiveness of the early initiation of VV-ECMO because it is a highly invasive procedure generally indicated for long-term use; therefore, its introduction should be carefully considered and systematically implemented. Under usual conditions involving patients with severe respiratory failure, mechanical ventilation is initiated after tracheal intubation, the patient is admitted to the intensive care unit and managed using prone positioning, neuromuscular blockade administration, and lung-protective ventilation strategies. VV-ECMO is introduced in cases of unimproved respiratory status despite the use of these treatments. However, this patient had acute and severe hypoxemia, which persisted and exacerbated to lethal hypoxemia even after mechanical ventilation. Therefore, VV-ECMO was urgently introduced in 
TA B L E 2. Ventilator parameters and arterial blood gas analyses.

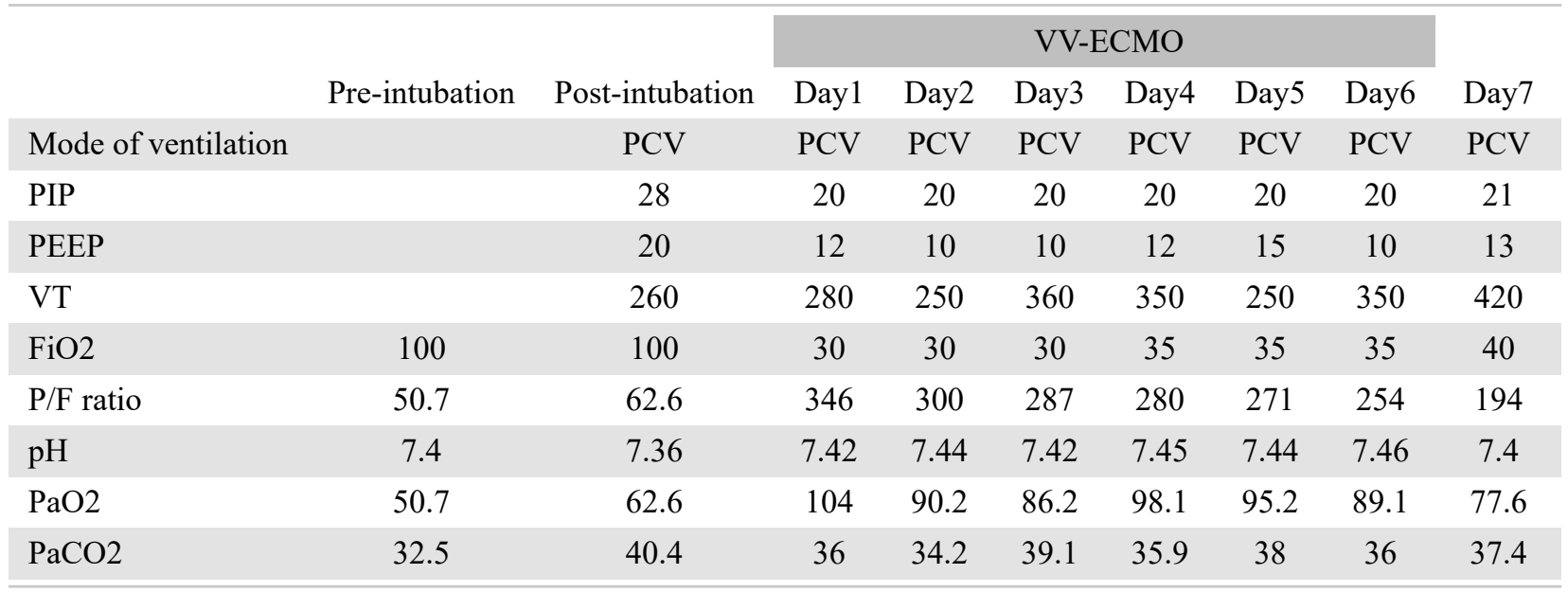

VV-ECMO: veno-venous extracorporeal membrane oxygenation; PCV: pressure control ventilation; PIP: peak inspiratory pressure $\left(\mathrm{cmH}_{2} \mathrm{O}\right)$; PEEP: positive end-expiratory pressure $\left(\mathrm{cmH}_{2} \mathrm{O}\right) ; \mathrm{VT}$ : tidal volume $\left(\mathrm{ml}^{2}\right.$; $\mathrm{FiO}_{2}$ : fraction of inspired oxygen (\%); $P / F$ ratio: ratio of partial pressure arterial oxygen and fraction of inspired oxygen ( $\mathrm{mm} \mathrm{Hg}$ ); $\mathrm{PaO}_{2}$ : partial pressure of oxygen in arterial blood ( $\mathrm{mm} \mathrm{Hg}$ ); $\mathrm{PaCO}_{2}$ : partial pressure of carbon dioxide in arterial blood ( $\mathrm{mm} \mathrm{Hg}$ ).

the ER as a life-saving procedure to prevent fatal hypoxemiainduced cardiac arrest. Subsequently, the ventilator was set as lung rest and VV-ECMO was removed on day 6 without complications. The advantages of introducing VV-ECMO in the hyperacute phase include prevention of hypoxemia, ensured gas exchange, and avoidance of subsequent lung injury. Fortunately, this patient was not exposed to subsequent hypoxemia, high airway pressure, or high oxygen concentration for a long time because of early VV-ECMO initiation. Therefore, the introduction of VV-ECMO earlier than previously recommended introduction protocols likely contributed to a life-saving and a favorable neurological prognosis.

In this case, prone positioning was not conducted before the introduction of VV-ECMO in the ER. Although prone positioning is not an indicated treatment with established efficacy, it possibly improved hypoxemia and prevented VVECMO introduction. However, prone positioning requires more personnel and time for its effectiveness; therefore, it was difficult to perform it in the ER.

VV-ECMO has been reported to be ineffective against COVID-19 because of the cytokine storm and hypercoagulation; furthermore, from the medical resources' viewpoint, the use of VV-ECMO should be restricted during the pandemic $[2,3]$. In addition, Zhou $\mathrm{F}$ et al. reported that COVID-19 had poor prognosis factors, such as old age, high sequential organ failure assessment (SOFA) score, and high D-Dimer levels ( $\geq 1.0 \mu \mathrm{g} / \mathrm{ml}$ ) [4]. However, in this case, the SOFA score was 6 and D-Dimer level was $3.0 \mu \mathrm{g} / \mathrm{ml}$. Although the prognosis is predicted to be poor with high SOFA scores and D-Dimer levels, the patient's life can still be saved via the early introduction of VV-ECMO and steroids, as observed in this case. In this case, steroids were administered before the administration of the antiviral drug (favipiravir), and respiratory conditions gradually improved after initiating steroid treatment, suggesting that steroid administration may have been effective. Certain reports have suggested improved clinical outcomes in patients with COVID-19 who were administered steroids $[5,6]$. In this case, methylprednisolone was administered. However, a recent report suggests that dexamethasone may reduce mortality of COVID-19 patients who are receiving respiratory support [7]. Thus, in the future, the administration of dexamethasone should be considered for patients with COIVD-19 who are receiving respiratory support.

\section{Conclusions}

For COVID-19 patients with severe ARDS, the introduction of VV-ECMO earlier than the conventional introduction standard may be effective. Further studies are required to evaluate the optimal timing of VV-ECMO for patients with COVID-19 and ARDS.

\section{ETHICS APPROVAL AND CONSENT TO PARTICIPATE}

This is a case report and does not need to be approved by the ethics committee.

\section{ACKNOWLEDGEMENTS}

We would like to thank all the peer reviewers and editors for their opinions and suggestions.

\section{FUNDING}

This research received no specific grant from any funding agency in the public, commercial, or not-for-profit sectors. 


\section{CONFLICT OF INTEREST}

The authors declare that there is no conflict of interest regarding the publication of this article.

\section{INFORMED CONSENT}

Informed consent was obtained from the patient. This article has been anonymized in accordance with the Act on the Protection of Personal Information.

\section{DATA AVAILABILITY}

The data used to support the findings of this study are available from the corresponding author upon request.

\section{REFERENCES}

[1] Hemmila MR, Rowe SA, Boules TN, et al. Extracorporeal life support for severe acute respiratory distress syndrome in adults. Ann Surg. 2004;240:595-605.

[2] Ramanathan K, Antognini D, Combes A, et al. Planning and provision of ECMO services for severe ARDS during the COVID-19 pandemic and other outbreaks of emerging infectious diseases. Lancet Respir Med. 2020;8:518-526.

[3] Zeng Y, Cai Z, Xianyu Y, et al. Prognosis when using extracorporeal membrane oxygenation (ECMO) for critically ill COVID-19 patients in China: a retrospective case series. Crit Care. 2020;24:148.

[4] Zhou F, Yu T, Du R, et al. Clinical course and risk factors for mortality of adult inpatients with COVID-19 in Wuhan, China: a retrospective cohort study. Lancet. 2020;395:1054-1062.

[5] Wu C, Chen X, Cai Y, et al. Risk factors associated with acute respiratory distress syndrome and death in patients with coronavirus disease 2019 pneumonia in Wuhan, China. JAMA Intern Med. 2020;180:934-943.

[6] The WHO Rapid Evidence Appraisal for COVID-19 Therapies (REACT) Working Group. Association between administration of systemic corticosteroids and mortality among critically ill patients with COVID-19: a meta-analysis. JAMA. 2020.

[7] The RECOVERY Collaborative Group. Dexamethasone in hospitalized patients with Covid-19- preliminary report. N Engl J Med. 2020. doi:10.1056/NEJMoa2021436.

How to cite this article: Kenta Aida, Kazunari Azuma, Kentaro Morinaga, Yuki Nishiyama, Kazuma Kimura, Jun Oda. Early Initiation of Veno-venous Extracorporeal Membrane Oxygenation in the Emergency Room as A Salvage Treatment for Patients with Coronavirus Disease 2019 with Severe Acute Respiratory Distress Syndrome. Signa Vitae. 2021;17(2):219-222. doi:10.22514/sv.2020.16.0102. 